\title{
Expression of hypoxia-inducible factor-1 $\alpha$ in synovial fluid and articular cartilage is associated with disease severity in knee osteoarthritis
}

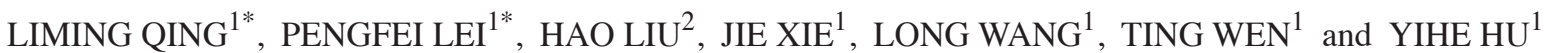 \\ ${ }^{1}$ Department of Orthopedic Surgery, Xiangya Hospital, Central South University, Changsha, Hunan 410008, P.R. China; \\ ${ }^{2}$ Program of Biology and Biomedical Sciences, Division of Medical Sciences, \\ Harvard Medical School, Harvard University, Boston, MA 02115, USA
}

Received July 21, 2015; Accepted September 9, 2016

DOI: 10.3892/etm.2016.3940

\begin{abstract}
The aim of the present study was to examine hypoxia-inducible factor $1 \alpha(\mathrm{HIF}-1 \alpha)$ levels in the synovial fluid and articular cartilage of patients with primary knee osteoarthritis (OA) and to investigate their association with the severity of disease. A total of 36 patients with knee OA and ten healthy controls were enrolled. Anteroposterior knee radiographs and/or Mankin scores were assessed to determine the disease severity of the affected knee. Radiographic grading of $\mathrm{OA}$ in the knee was performed according to Kellgren-Lawrence criteria. HIF-1 $\alpha$ levels in synovial fluid were measured using enzyme-linked immunosorbent assay, whereas HIF-1 $\alpha$ levels in articular cartilage were assessed with immunohistochemical methods. Compared with healthy controls, OA patients exhibited an increased HIF-1 $\alpha$ concentration in synovial fluid $(218.17 \pm 25.12$ vs. $156.66 \pm 7.74 \mathrm{pg} / \mathrm{ml}$; $\mathrm{P}<0.001)$ and articular cartilage $(\mathrm{P}<0.05)$. Furthermore, synovial fluid HIF-1 $\alpha$ levels demonstrated a positive correlation with articular cartilage HIF-1 $\alpha$ levels (Pearson's $\mathrm{P}=0.815$; $\mathrm{P}<0.001)$. Subsequent analysis showed that synovial fluid HIF-1 $\alpha$ levels were significantly correlated with the severity of disease (Spearman's $\rho=0.933 ; \mathrm{P}<0.001)$. Furthermore, articular cartilage levels of HIF-1 $\alpha$ also correlated with disease severity (Spearman's $\rho=-0.967 ; \mathrm{P}<0.001$ ). The findings of the present study suggested that HIF-1 $\alpha$ in synovial fluid and articular cartilage is associated with progressive joint damage and is likely to be a useful biomarker for determining disease severity and progression in knee OA.
\end{abstract}

Correspondence to: Professor Yihe Hu, Department of Orthopedic Surgery, Xiangya Hospital, Central South University, 87 Xiangya Road, Changsha, Hunan 410008, P.R. China

E-mail: csuhuyihe@163.com

* Contributed equally

Key words: hypoxia-inducible factor $1 \alpha$, osteoarthritis, synovial fluid, articular cartilage

\section{Introduction}

Osteoarthritis (OA), which is an age-related condition, is the leading cause of pain, disability and shortening of adult working life (1). Incidence of OA increases with age, with $25 \%$ of individuals aged $>50$ years old exhibiting OA of the knee. Worldwide, $\sim 10 \%$ of men, $18 \%$ of women, and $60-65 \%$ of individuals aged over 60 years have symptomatic OA, and $80 \%$ of these patients suffer from limitations in motion $(2,3)$. Clinical symptoms of OA include stiffness, pain, limited motion, crepitus, swelling and deformity $(4,5)$. Despite promising preclinical data covering various molecule classes, the etiology of OA remains poorly elucidated (4). Several biochemical and biomechanical factors have been reported and are considered to be potential lines of investigation for the pathogenesis of OA $(5,6)$.

Hypoxia-inducible factor $1 \alpha(\mathrm{HIF}-1 \alpha)$ is considered to be a key regulator of cellular adaptation to hypoxic conditions and catabolic stress through the activation of hypoxic response elements (7-9). $\mathrm{O}_{2}$-dependent binding of the von Hippel-Lindau tumor suppressor protein ensures it is able to associate with HIF-1 $\alpha$ for proteasomal degradation and ubiquitination. The activity of the HIF-1 $\alpha$ transactivation domains also depends on $\mathrm{O}_{2}$ regulation through a previously undefined mechanism. Articular cartilage is known to physiologically lack blood vessels, which results in a significantly decreased oxygen level within the tissue; thus, the resident cells require well-adapted mechanisms to ensure survival. Previous studies have demonstrated that HIF-1 $\alpha$ is associated with the regulation of anaerobic energy generation, glucose transport and matrix synthesis by articular chondrocytes (10-12). Furthermore, proinflammatory mediators and mechanical load have also been suggested to increase HIF-1 $\alpha$ activity in articular chondrocytes (13-15). These factors are known to be associated with the pathogenesis of OA. Therefore, it is a reasonable assumption that osteoarthritis chondrocytes depend on HIF-1 $\alpha$ to survive and function properly.

HIF-1 $\alpha$ transcription factor also has an important role in maintaining proper cellular functions under hypoxic conditions $(16,17)$. Grimmer et al (18) have revealed that HIF- $1 \alpha$ is associated with the upregulation of microsomal 
prostaglandin E synthase-1 and has an important role in the metabolism of OA cartilage.

Although articular cartilage and/or synovial fluid levels of several cytokines have previously been investigated in patients with knee OA (4-6); to the best of our knowledge, there have been no detailed studies on articular cartilage and synovial fluid levels of HIF-1 $\alpha$ in various clinical stages of primary knee OA. The association between HIF-1 $\alpha$ levels in articular cartilage and disease severity has not been previously reported in the literature. The purpose of the present study was to investigate the expression levels of HIF-1 $\alpha$ in the articular cartilage and synovial fluid of patients with primary knee OA, and evaluate the potential correlation with the osteoarthritic disease process via Mankin scoring and radiographic grading of knee OA, and to further elucidate the pathways associated with the progression of $\mathrm{OA}$.

\section{Materials and methods}

Patients and sample preparation. The present study was approved by the Ethical Committee of Xiangya Hospital Central South University (Changsha, China), and was conducted in accordance with the Declaration of Helsinki. A total of 36 patients with primary knee OA (11 males and 25 females; mean age 67.4 years), diagnosed according to the criteria of the American College of Rheumatology, and 10 normal healthy individuals (4 males and 6 females; mean age 58.9 years) were enrolled. Data were reviewed to exclude any forms of secondary OA and inflammatory-associated joint diseases, such as rheumatoid arthritis (RA). Table I shows the patient characteristics.

Disease severity was evaluated with radiographs of the affected knee according to the Kellgren and Lawrence (KL) classification (19). A total of 36 osteoarthritic cartilage and synovial tissue samples were harvested from 36 patients who underwent a total knee replacement due to primary knee OA. Normal cartilage and synovial tissue was collected from 10 human knees at the time of autopsy. Osteoarthritic changes were classified histomorphologically, using modified Mankin scoring (20) as follows: Normal, 0-1; mild lesions, 2-5; moderate lesions, 6-9; and severe lesions, 10-14. The following samples were included in the present study: 10 samples (Mankin score 0-1), 8 samples (Mankin score 2-5), 13 samples (Mankin score 6-9) and 15 samples (Mankin score 10-14).

Immunohistochemistry. Specimens were immediately fixed with $4 \%$ paraformaldehyde in PBS, decalcified with $0.2 \mathrm{M}$ ethylenediaminetetraacetid acid, embedded in paraffin wax and cut into $5-\mu \mathrm{M}$-thick sections. Primary monoclonal antibodies against HIF-1 $\alpha$ (1:10,000; cat. no. NB100-105; Novus Biologicals, LLC, Littleton, CO, USA) were incubated with the specimens overnight at $4^{\circ} \mathrm{C}$ for immunohistochemical analyses. A catalyzed signal amplification kit (DakoCytomation, Carpinteria, CA, USA) based on a streptavidin-biotin peroxidase reaction was employed for the visualization of HIF-1 $\alpha$, with diaminobenzidine as a chromogen. Control sections were incubated with nonimmune goat antisera (Zhongshan Golden Bridge Biotechnology Co., Ltd., Beijing, China). Sections were examined under a microscope at 100x magnification to evaluate the expression of HIF-1 $\alpha$. Relative HIF-1 $\alpha$ expression
Table I. Patient characteristics.

\begin{tabular}{lcc}
\hline Variable & Osteoarthritis & Normal \\
\hline Number of patients & 36 & 10 \\
Age range (years) & $54-85$ & $54-63$ \\
Mean age (years) & 67.4 & 58.9 \\
Sex & & \\
Male & 11 & 4 \\
Female & 25 & 6 \\
\hline
\end{tabular}

levels in articular cartilage were quantified and visualized as gray values. MIAS-4400 ImageJ (National Institutes of Health, Bethesda, MA, USA) software was used to perform semiquantitative assessment of the mean gray values of HIF-1 $\alpha$ expression. All the sections were evaluated by a pathologist who was blinded to the clinical data. All densities were normalized to PBS and repeated in triplicate. A total of three sections per sample were measured and the mean was calculated to reduce the error arising from the variations in section thickness. The final data consisted of a mean of three independent measurements representing the mean levels of $\mathrm{HIF}-1 \alpha$ in the articular cartilage samples.

Enzyme-linked immunosorbent assay (ELISA). During total knee replacement surgery, synovial fluid was aspirated from the affected knee, and immediately centrifuged at 1,000 x $g$ for $25 \mathrm{~min}$ at $4^{\circ} \mathrm{C}$ to remove joint debris and cells. According to the manufacturers' instructions, the expression of HIF-1 $\alpha$ in synovial fluid was detected using a commercial ELISA kit (Uscn Life Science, Inc., Wuhan, China). The assays had inter-assay coefficients of variation that were $<6 \%$ and intra-assay coefficients of variation of $<5 \%$. Two independent experiments were performed.

Statistical analysis. SPSS for Windows (version 17.0; SPSS, Inc., Waltham, MA, USA) was used for statistical analyses. Student's t-test was used to compare the means of two independent groups and one way analysis of variance was used to compare the means of $>2$ independent groups, followed by Student-Newman-Keul (SNK) test when comparing among the groups. Spearman's correlation was used to evaluate the correlation between synovial fluid levels of HIF-1 $\alpha$ and the severity of OA. Pearson's correlation and linear regression analysis were employed to determine the correlation between synovial fluid HIF-1 $\alpha$ expression levels and the gray values of HIF-1 $\alpha$ in articular cartilage. Spearman's correlation and linear regression analysis were applied to evaluate the correlation between the gray values of HIF-1 $\alpha$ in articular cartilage and the Mankin score of OA. All data were expressed as the mean \pm standard deviation. $\mathrm{P}<0.05$ was considered to indicate a statistically significant difference.

\section{Results}

HIF-1 $\alpha$ expression levels in articular cartilage. A total of 46 knees were evaluated from 10 controls and 36 patients. HIF-1 $\alpha$ expression levels were detected in the tissues of all the 
four groups, which were categorized according to the severity of the lesions in the cartilage (Fig. 1). The average gray value of HIF-1 $\alpha$ expression was $205.49 \pm 4.95$ in normal cartilage, $185.34 \pm 9.09$ in cartilage with mild lesions, $171.26 \pm 3.40$ in cartilage with moderate lesions and $155.48 \pm 10.41$ in cartilage with severe lesions, respectively (Table II). Significant differences in the average gray values of HIF-1 $\alpha$ expression were detected among the groups $(\mathrm{P}<0.05)$. The average gray value of HIF-1 $\alpha$ expression in each group was demonstrated to be correlated with disease severity according to the modified Mankin score (Spearman's $\rho=-0.967, \mathrm{P}<0.001$ ) (Fig. 2).

HIF-1 $\alpha$ expression levels in synovial fluid. The concentrations of HIF-1 $\alpha$ in the synovial fluid of patients with knee $\mathrm{OA}$ are demonstrated in Fig. 3. OA patients exhibited higher HIF- $1 \alpha$ concentrations compared with the healthy controls (218.17 \pm 25.12 vs. $156.66 \pm 7.74$ pg/ml, P<0.001). Synovial fluid concentrations of HIF-1 $\alpha$ were compared and analyzed in relation to the radiological KL grading values of OA. The concentrations of HIF- $1 \alpha$ in the synovial fluid of KL grade 2 were $179.91 \pm 12.49 \mathrm{pg} / \mathrm{ml}$ [95\% confidence interval (CI), 169.47-190.36], those from KL grade 3 were $216.37 \pm 9.51 \mathrm{pg} / \mathrm{ml}$ (95\% CI, 210.62-222.12), whereas those from KL grade 4 were $240.14 \pm 8.16 \mathrm{pg} / \mathrm{ml}(95 \% \mathrm{CI}, 235.62-244.66)$. The data indicated that synovial fluid levels of HIF-1 $\alpha$ in cartilage graded as KL grade 4 were significantly increased compared with those of KL grade 2 and $3(\mathrm{P}<0.01)$. The levels of HIF-1 $\alpha$ also correlated with the severity of disease (Spearman's $\rho=0.933$, $\mathrm{P}<0.001$; Fig. 4). Notably, synovial fluid HIF-1 $\alpha$ concentrations exhibited a significant correlation with articular cartilage HIF-1 $\alpha$ expression levels (Pearson's $\rho=-0.815$; $\mathrm{P}<0.001$; Fig. 5).

\section{Discussion}

To the best of our knowledge, the present study is the first to evaluate the levels of HIF-1 $\alpha$ in articular cartilage and synovial fluid and its correlation with the severity of knee OA disease. The present findings demonstrated a marked increase in HIF-1 $\alpha$ levels in the articular cartilage and synovial fluid of patients with knee OA compared with the controls. Previous studies have indicated that human and bovine articular chondrocytes and murine epiphyseal chondrocytes express HIF-1 $\alpha$ (21-23). HIF-1 $\alpha$ in the synovial fluid is thought to have originated from local tissues, such as the synovial membrane and articular cartilage. Numerous studies have demonstrated that HIF-1 $\alpha$ is a key factor that influences articular chondrocyte behavior during cartilage homeostasis and osteoarthritis (22-24). HIF-1 $\alpha$ is a highly conserved transcription factor that has important functions in the control of energy generation, matrix synthesis and cell survival by articular and growth-plate chondrocytes $(25,26)$. Previous studies have revealed that the stabilization of HIF-1a may be a potential tool for increasing cell vitality, matrix synthesis and cartilage integrity in patients with osteoarthritis (24-26). Notably, synovial fluid HIF-1 $\alpha$ concentrations exhibited a correlation with articular cartilage HIF-1 $\alpha$ expression levels in the present study. Therefore, detecting the levels of HIF-1 $\alpha$ in synovial fluid may be used as a marker to predict the degree of cartilaginous damage and disease severity.
Table II. Mean gray value of HIF-1 $\alpha$ expression.

\begin{tabular}{lcc}
\hline Group & No. of samples & $\begin{array}{c}\text { Average gray value } \\
\text { of HIF-1 } \alpha \text { expression }\end{array}$ \\
\hline Normal & 10 & $205.49 \pm 4.95$ \\
Mild lesions & 8 & $185.34 \pm 9.09$ \\
Moderate lesions & 13 & $171.26 \pm 3.40$ \\
Severe lesions & 15 & $155.48 \pm 10.41$ \\
\hline
\end{tabular}

Data are presented as the mean \pm standard deviation. Difference in the average gray value of HIF- $1 \alpha$ expression levels in the cartilage of the different groups indicated statistically significance when compared between each group $(\mathrm{P}<0.05)$. HIF-1 $\alpha$, hypoxia-inducible factor $1 \alpha$.

HIF-1 $\alpha$ is degraded by an $\mathrm{O}_{2}$-dependent mechanism. Under normoxic conditions, HIF-1 $\alpha$ is hydroxylated to allow binding of the von Hippel-Lindau tumor suppressor protein to HIF-1 $\alpha$, and this binding triggers subsequent enzymatic degradation. Under hypoxic conditions $\left(<5 \% \mathrm{O}_{2}\right)$, HIF-1 $\alpha$ is stable and can be detected (8). HIF-1 $\alpha$ localizes into the nucleus to associate with HIF-1 $\beta$ to form a heterodimer and activate hypoxia-inducible target genes (13). Hypoxia has previously been demonstrated to increase matrix synthesis of epiphyseal chondrocytes (27), and to induce vascular endothelial growth factor (VEGF) expression in normal chondrocytes via HIF-1 $\alpha$ activity $(28,29)$. VEGF is a key angiogenesis factors, which is able to thicken the synovial membrane, deposit new extracellular matrix and increase the proliferation of synovial fibroblasts. All those factors are essential for the development of OA. The importance of angiogenesis in OA has been further elucidated by several authors in recent publications that demonstrated that new blood vessels are not only formed in synovium but also in other tissues $(30,31)$. The articular cartilage of patients with OA is invaded at the osteochondral junction by blood vessels from the subchondral bone, which subsequently induces an increase in blood vessel density in the non-calcified cartilage (30).

HIF-1 $\alpha$ also is a potent transactivator of numerous genes, including various MMPs (MMP2, 3, 9 and 13) (15) and heat shock protein (28), which are known to act as cellular chaperones for proteins that are misfolded under conditions of cellular stress. Therefore, we hypothesize that $\mathrm{HIF}-1 \alpha$ has an important role in $\mathrm{O}_{2}$-dependent signaling pathways in the articular chondrocytes, as supported by previous research (32). Therefore, elevated HIF-1 $\alpha$ levels may be associated with the development of osteoarthritis.

The role of oxygen as an important modulator of gene expression is well-recognized and extensive evidence has indicated that HIF is the primary gene and regulatory factor that responds to key variations in $\mathrm{O}_{2}$ levels $(7,8)$. Genes regulated at the transcription level by HIF-1 $\alpha$ are associated with numerous cellular functional events, including angiogenesis, vascular reactivity and remodeling (9), vasomotor control, glucose and energy metabolism (26), erythropoiesis, iron homeostasis, $\mathrm{pH}$ regulation, cell proliferation and 
A

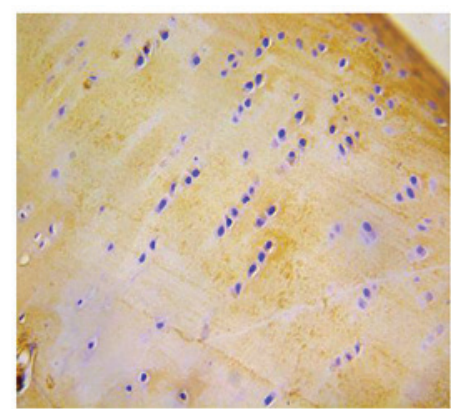

C

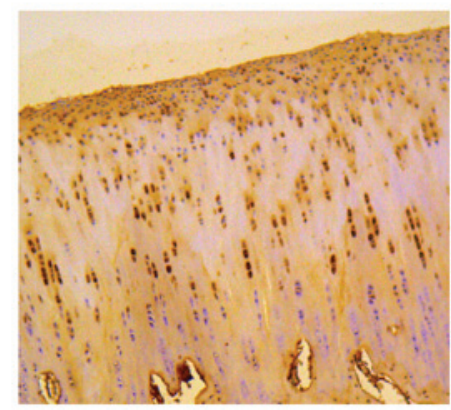

B

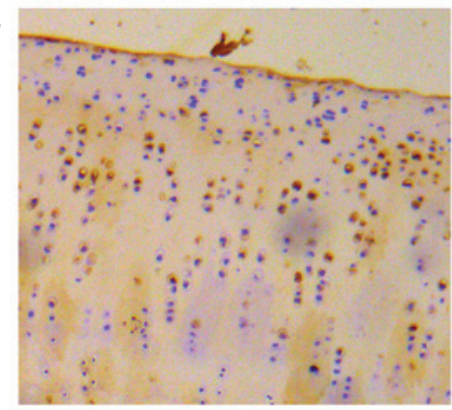

D

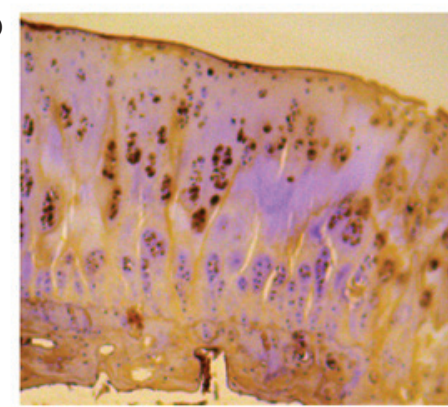

Figure 1. Hypoxia-inducible factor $1 \alpha$ immunohistochemical staining in the articular cartilage of knee osteoarthritis patients and controls demonstrated (A) normal cartilage, (B) mildly leisoned cartilage, (C) moderately leisoned cartilage (D) and severely leisoned cartilage. Magnification, x200.

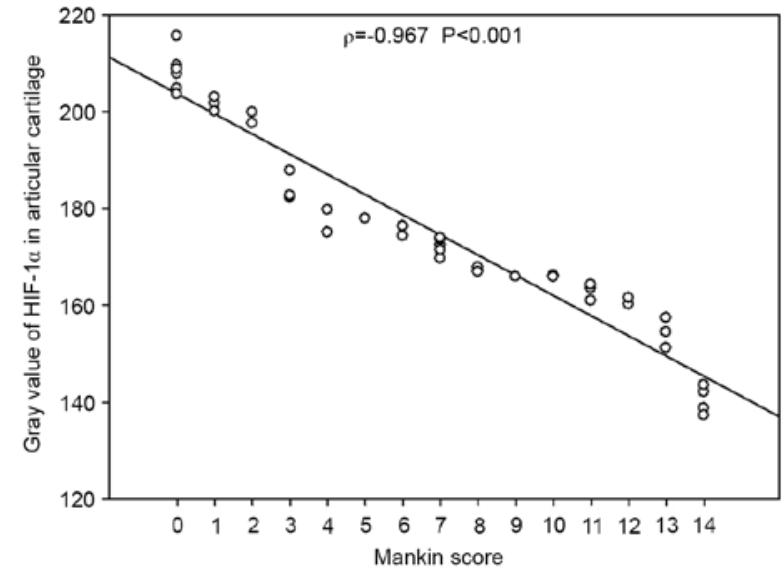

- Mankin score vs gray value of HIF- $1 \alpha$ in articular cartilage

Figure 2. Gray value of HIF-1 $\alpha$ in articular cartilage is correlated with Mankin score of osteoarthritis (Spearman's $\rho=-0.967 ; \mathrm{P}<0.001$ ). Osteoarthritis changes were classified histomorphologically, using the Mankin grading system. Spearman's correlation and linear regression analyses were used to determine whether the gray value of HIF-1 $\alpha$ in articular cartilage correlated with the Mankin score of osteoarthritis. HIF-1 $\alpha$, hypoxia-inducible factor $1 \alpha$.

viability, nucleotide metabolism, matrix metabolism, and metal transport $(29,30)$. Furthermore, HIF-1 $\alpha$ is essential for chondrogenesis, as it is associated with chondrocyte growth arrest, survival, maturation, and apoptosis (33-35). HIF-1 $\alpha$ also regulates the configuration and maintenance of articular cartilage via the induction of anabolic factors and the suppression of key catabolic factors (36). The migration and invasion of fibroblast-like synoviocytes (FLSs) have also been demonstrated to be critical for the pathogenesis of OA $(37,38)$. Li et al (15) showed that HIF-1 $\alpha$ levels contribute to the migration and invasion of FLSs by upregulating the

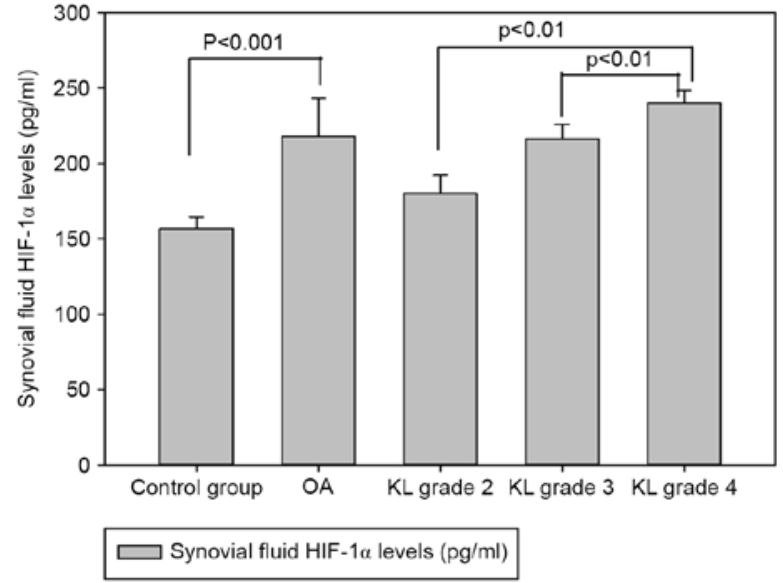

Figure 3. Synovial fluid levels of HIF-1 $\alpha$ in healthy controls $(n=10)$, osteoarthritis patients $(n=36)$ and osteoarthritis patients with KL grade $2(n=8), K L$ grade $3(n=13)$ and KL grade $4(n=15)$. HIF-1 $\alpha$, hypoxia-inducible factor $1 \alpha$; KL, Kellgren and Lawrence; OA, osteoarthritis.

expression of MMP2 and MMP9 via the activation of the $\mathrm{NF}-\kappa \mathrm{B} / \mathrm{HIF}-1 \alpha$ signalling pathway. These findings indicated that levels of HIF-1 $\alpha$ in both synovial fluid and cartilage may play an important role in the pathogenesis of OA.

There are several potential limitations to the present study. Firstly, the sample size was not large enough to achieve definitive conclusions. Secondly, only those patients who attended Xiangya Hospital for treatment of knee OA were investigated. Thirdly, the cross-sectional design of the study precluded addressing whether the analyzed level of HIF-1 $\alpha$ predicted alterations in the severity of knee OA.

In conclusion, patients with knee OA exhibited elevated levels of HIF-1 $\alpha$ compared with healthy controls in synovial fluid. Furthermore, the expression of HIF-1 $\alpha$ in articular cartilage and synovial fluid was significantly correlated with 


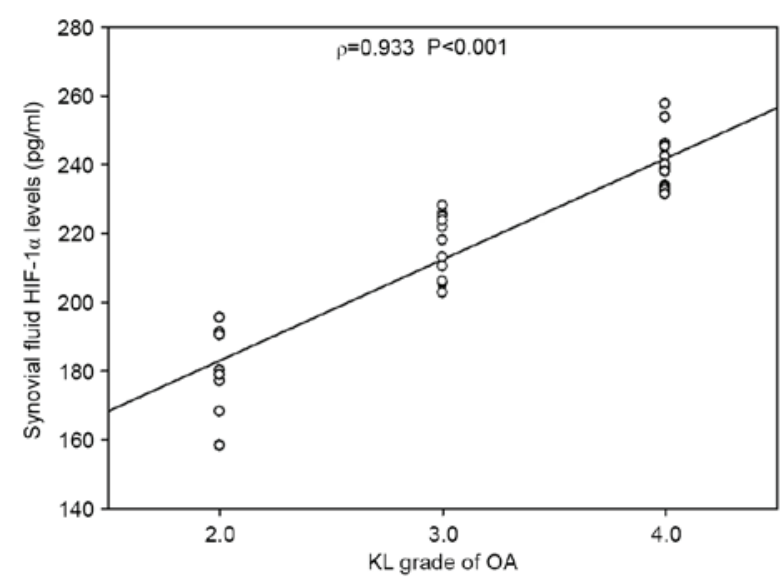

- $\mathrm{KL}$ grade vs synovial fluid HIF- $1 \alpha$ levels $(\mathrm{pg})$ - Plot 1 Regr

Figure 4. Synovial fluid levels of HIF-1 $\alpha$ are correlated with the KL grade of osteoarthritis (Spearman's $\rho=0.933$; $\mathrm{P}<0.001$ ). Disease severity was determined via weight-bearing anteroposterior radiographs of the affected knee. Knee radiographs were evaluated according to the KL classification. Spearman's correlation was employed to determine the correlation between synovial fluid levels of HIF-1 $\alpha$ and severity of osteoarthritis. HIF-1 $\alpha$, hypoxia-inducible factor $1 \alpha$; KL, Kellgren and Lawrence; OA, osteoarthritis.

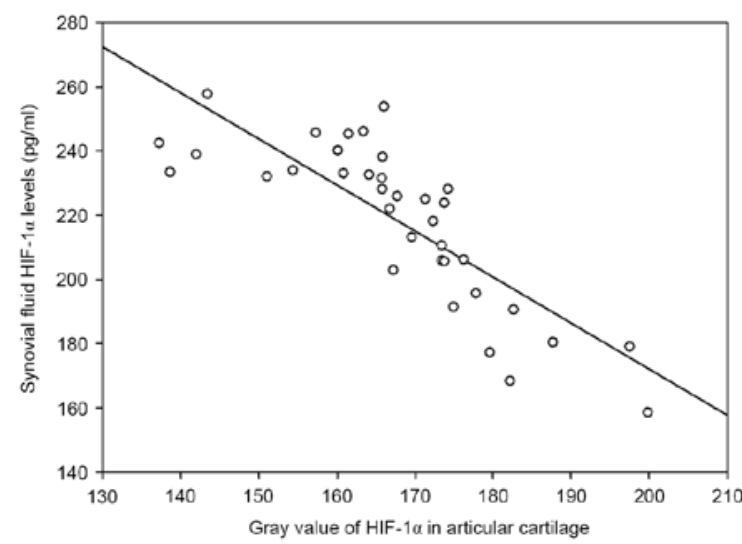

- Gray value of HIF-1a in articular cartilage vs synovial fluid HIF-1 $\alpha$ levels - Plot 1 Regr

Figure 5. Synovial fluid HIF-1 $\alpha$ levels are correlated with gray value of HIF-1 $\alpha$ in articular cartilage (Pearson's $\rho=-0.815 ; \mathrm{P}<0.001)$. Pearson's correlation and linear regression analyses were applied to determine the correlation between synovial fluid HIF-1 $\alpha$ levels and the gray values of HIF-1 $\alpha$ in articular cartilage. HIF-1 $\alpha$, hypoxia-inducible factor $1 \alpha$.

the severity disease in knee OA. Further studies are required to elucidate the contribution of HIF-1 $\alpha$ to the pathogenesis of OA.

\section{Acknowledgments}

This study was supported by grants from the National Natural Science Foundation of China (grant no. 81371934), the Open-End Fund for the Valuable and Precision Instruments of Central South University (grant no. CSUZC2014046) and Hunan Provincial Innovation Foundation For Postgraduates (grant no. CX2014B111).

\section{References}

1. Gallo J, Goodman SB, Konttinen YT, Wimmer MA and Holinka M: Osteolysis around total knee arthroplasty: A review of pathogenetic mechanisms. Acta Biomaterialia 9: 8046-8058, 2013.

2. van den Berg WB: Osteoarthritis yearn review: Pathomechanisms. Osteoarthritis Cartilage 19: 338-341, 2011.

3. Saito T and Kawaguchi H: HIF-2 $\alpha$ as a possible therapeutic target of osteoarthritis. Osteoarthritis Cartilag 18: 1552-1556, 2010.

4. Lotz M: Osteoarthritis yearn review: Biology. Osteoarthritis Cartilage 20: 192-196, 2012.

5. Tonge DP, Pearson MJ and Jones SW: The hallmarks of osteoarthritis and the potential to develop personalised disease-modifying pharmacological therapeutics. Osteoarthritis Cartilage 22: 609-621, 2014.

6. Alcaraz MJ, Megías J, García-Arnandis I, Clérigues V and Guillén MI: New molecular targets for the treatment of osteoarthritis. Biochem Pharmacol 80: 13-21, 2010.

7. Araldi E and Schipani E: Hypoxia, HIFs and bone development. Bone 47: 190-196, 2010.

8. Coimbra IB, Jimenez SA, Hawkins DF, Piera-Velazquez S and Stokes DG: Hypoxia inducible factor-1 alpha expression in human normal and osteoarthritic chondrocytes. Osteoarthritis Cartilage 12: 336-345, 2004.

9. Cramer T, Schipani E, Johnson RS, Swoboda B and Pfander D: Expression of VEGF isoforms by epiphyseal chondrocytes during low-oxygen tension is HIF-1alpha dependent. Osteoarthritis Cartilage 12: 433-439, 2004.

10. Duval E, Baugé C, Andriamanalijaona R, Bénateau H, Leclercq S Dutoit S, Poulain L, Galéra P and Boumédiene K: Molecular mechanism of hypoxia-induced chondrogenesis and its application in in vivo cartilage tissue engineering. Biomaterialsm 33: 6042-6051, 2012

11. Gelse K, Mühle C, Knaup K, Swoboda B, Wiesener M, Henning F, Olk A and Schneider H: Chondrogenic differentiation of growth factor-stimulated precursor cells in cartilage repair tissue is associated with increased HIF-1alpha activity. Osteoarthritis Cartilage 16: 1457-1465, 2008.

12. Hong YH, Park CW, Kim HS, Won KC, Kim YW and Lee CK: Effects of hypoxia/ischemia on catabolic mediators of cartilage in a human chondrocyte, SW1353. Biochem Biophys Res Commun 431: 478-483, 2013.

13. Hatta TK, Kishimoto KN, Okuno H and Itoi E: Lubricin expression is suppressed by hypoxia through HIF-1 mediated pathway. Osteoarthritis Cartilage 20: S137-S138, 2012.

14. Henrotin Y, Kurz B and Aigner T: Oxygen and reactive oxygen species in cartilage degradation: Friends or foes? Osteoarthritis Cartilage 13: 643-654, 2005.

15. Li G, Zhang Y, Qian Y, Zhang H, Guo S, Sunagawa M, Hisamitsu T and Liu Y: Interleukin-17A promotes rheumatoid arthritis synoviocytes migration and invasion under hypoxia by increasing MMP2 and MMP9 expression through NF- $\kappa \mathrm{B} / \mathrm{HIF}-1 \alpha$ pathway. Mol Immunol 53: 227-236, 2013.

16. Koh MY and Powis G: Passing the baton: the HIF switch. Trends Biochem Sci 37: 364-372, 2012.

17. Zhang C, Yang F, Cornelia R, Tang W, Swisher S and Kim H: Hypoxia-inducible factor-1 is a positive regulator of Sox 9 activity in femoral head osteonecrosis. Bone 48: 507-513, 2011.

18. Grimmer C, Balbus N, Lang U, Aigner T, Cramer T, Müller L, Swoboda B and Pfander D: Regulation of Type II collagen synthesis during osteoarthritis by prolyl-4-hydroxylases: Possible influence of low oxygen levels. Am J Pathol 169: 491-502, 2006.

19. Kellgren JH and Lawrence JS: Radiological assessment of osteo-arthrosis. Ann Rheum Dis 16: 494-502, 1957.

20. Van der Sluijs JA, Geesink RG, Van der Linden AJ, Bulstra SK, Kuyer R and Drukker J: The reliability of the Mankin scores for osteoarthritis. J Orthop Res 10: 58-61, 1992.

21. Zhang RX, Ren K and Dubner R: Osteoarthritis pain mechanisms: Basic studies in animal models. Osteoarthritis Cartilage 21: 1308-1315, 2013.

22. Meretoja VV, Dahlin RL, Wright S, Kasper FK and Mikos SG: The effect of hypoxia on the chondrogenic differentiation of co-cultured articular chondrocytes and mesenchymal stem cells in scaffolds. Biomaterials 34: 4266-4273, 2013.

23. Nomura T, Aoyama M, Waguri-Nagaya Y, Goto Y, Suzuki M, Miyazawa K, Asai K and Goto S: Tumor necrosis factor stimulates osteoclastogenesis from human bone marrow cells under hypoxic conditions. Exp Cell Res 321: 167-177, 2014. 
24. Lin JL, Wang MJ, Lee DC, Liang CC and Lin SK: Hypoxia-inducible factor-1alpha regulates matrix metalloproteinase-1 activity in human bone marrow-derived mesenchymal stem cells. FEBS Lett 582: 2615-2619, 2008.

25. Makris EA, Hu JC and Athanasiou KA: Hypoxia-induced collagen crosslinking as a mechanism for enhancing mechanical properties of engineered articular cartilage. Osteoarthritis Cartilage 21: 634-641, 2013

26. Ren BF, Deng LF, Wang J, Zhu YP, Wei L and Zhou Q: Hypoxia regulation of facilitated glucose transporter-1 and glucose transporter-3 in mouse chondrocytes mediated by HIF-1alpha. Joint Bone Spine 75: 176-181, 2008.

27. Murata M, Yudoh K and Masuko K: The potential role of vascular endothelial growth factor (VEGF) in cartilage: How the angiogenic factor could be involved in the pathogenesis of osteoarthritis? Osteoarthritis Cartilage 16: 279-286, 2008.

28. Zhu G, Tang Y, Liang X, Zheng M, Yang J, Zhou H, Li L and Qin T: Role of hypoxia-inducible factor-1 alpha in the regulation of plasminogen activator activity in rat knee joint chondrocytes. Osteoarthritis Cartilage 17: 1494-1502, 2009.

29. Zhu M, Feng Q and Bian LM: Differential effect of hypoxia on human mesenchymal stem cell chondrogenesis and hypertrophy in hyaluronic acid hydrogels. Acta Biomater 10: 1333-1340, 2014.

30. Chim SM, Tickner J, Chow ST, Kuek V, Guo BS, Zhang G, Rosen V, Erber W and Xu JK: Angiogenic factors in bone local environment. Cytokine Growth Factor Rev 24: 297-310, 2013.

31. Matsuki TY, Arai Y, Tsuchida S, Terauchi R, Inoue H, Nakagawa S, Inoue $\mathrm{A}$, Mazda $\mathrm{O}$ and Kubo $\mathrm{T}$ : The roles of heat shock protein 70 on chondrocyte. Osteoarthritis Cartilage 21: S127, 2013.

32. Pfander D, Swobodal B and Cramer T: The role of HIF-1alpha in maintaining cartilage homeostasis and during the pathogenesis of osteoarthritis. Arthritis Res Ther 8: 104, 2006.
33. Neve A, Cantatore FP, Corrado A, Gaudio A, Ruggieri S and Ribatti D: In vitro and in vivo angiogenic activity of osteoarthritic and osteoporotic osteoblasts is modulated by VEGF and vitamin D3 treatment. Regul Pept 184: 81-84, 2013.

34. Pufe T, Lemke A, Kurz B, Petersen W, Tillmann B, Grodzinsky AJ and Mentlerin R: Mechanical overload induces VEGF in cartilage discs via hypoxia-inducible factor. Am J Pathol 164: 185-192, 2004.

35. Okada KY, Hosaka Y, Kobayashi H, Sugita S, Chang S, Mori Y, Akiyama H, Tanaka S, Kawaguchi H and Saito T: HIF-1a regulates configuration and maintenance of articular cartilage through induction of anabolic factors and suppression of catabolic factors. Osteoarthritis Cartilage 22: S164-S165, 2014.

36. Sakamoto J, Origuchi T, Okita M, Nakano J, Kato K, Yoshimura T, Izumi S, Komori T, Nakamura H, Ida $\mathrm{H}$, et al: Immobilization-induced cartilage degeneration mediated through expression of hypoxia-inducible factor-1alpha, vascular endothelial growth factor, and chondromodulin-I. Connect Tissue Res 50: 37-45, 2009.

37. Zhang C, Wei X, Chen C, Cao K, Li Y, Jiao Q, Ding J, Zhou J, Fleming $\mathrm{BC}$, Chen $\mathrm{Q}$, et al: Indian hedgehog in synovial fluid is a novel marker for early cartilage lesions in human knee joint. Int J Mol Sci 15: 7250-7265, 2014.

38. Walsh DA, Bonnet CS, Turner EL, Wilson D, Situ M and Mcwilliams SF: Angiogenesis in the synovium and at the osteochondral junction in osteoarthritis. Osteoarthritis Cartilage 15: 743-751, 2007. 